\title{
Transmission low-energy electron diffraction using double-gated single nanotip field emitter
}

Chiwon Lee, Soichiro Tsujino, and R. J. Dwayne Miller

Citation: Appl. Phys. Lett. 113, 013505 (2018); doi: 10.1063/1.5030889

View online: https://doi.org/10.1063/1.5030889

View Table of Contents: http://aip.scitation.org/toc/apl/113/1

Published by the American Institute of Physics

\section{Articles you may be interested in}

Dielectric-induced interface states in black phosphorus and tungsten diselenide capacitors

Applied Physics Letters 113, 013103 (2018); 10.1063/1.5040093

Ultrathin amorphous $\mathrm{ZnGe}_{\mathrm{X}} \mathrm{SnO}$ films for high performance ultra-thin-film transistors

Applied Physics Letters 113, 013504 (2018); 10.1063/1.5031448

Control of dipole properties in high-k and $\mathrm{SiO}_{2}$ stacks on $\mathrm{Si}$ substrates with tricolor superstructure

Applied Physics Letters 113, 012103 (2018); 10.1063/1.5034494

Multi-color and multidirectional-steerable Smith-Purcell radiation from 2D sub-wavelength hole arrays

Applied Physics Letters 113, 013501 (2018); 10.1063/1.5034248

Artificial electronic synapse characteristics of a Ta/Ta2 $\mathrm{O}_{5-\mathrm{x}} / \mathrm{Al}_{2} \mathrm{O}_{3} / \mathrm{InGaZnO}_{4}$ memristor device on flexible stainless steel substrate

Applied Physics Letters 113, 013503 (2018); 10.1063/1.5027776

Piezoelectric graphene field effect transistor pressure sensors for tactile sensing

Applied Physics Letters 113, 014102 (2018); 10.1063/1.5030545

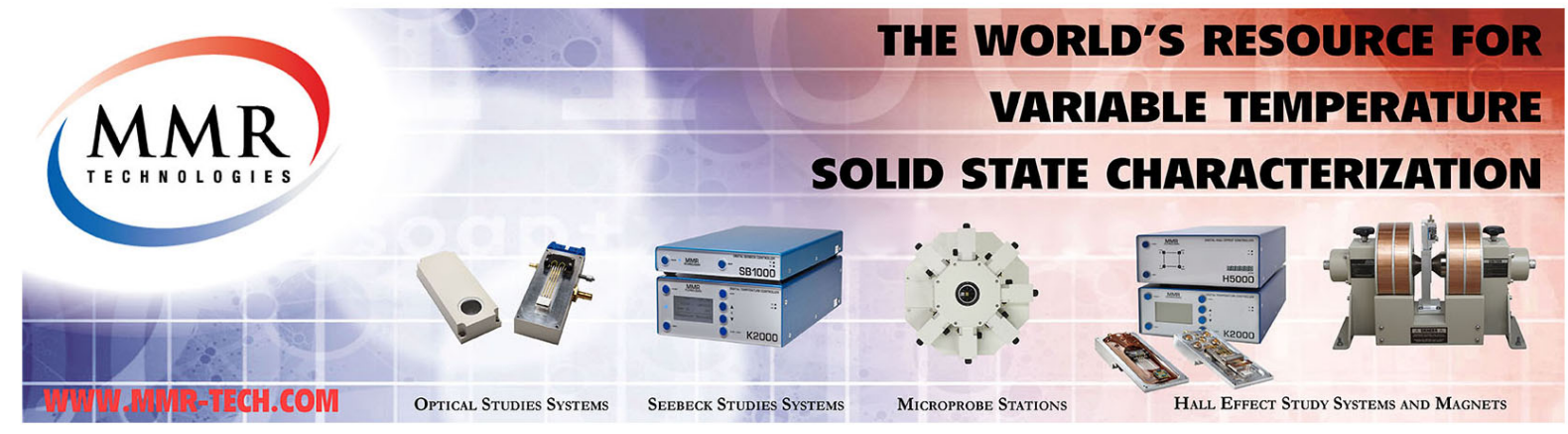




\title{
Transmission low-energy electron diffraction using double-gated single nanotip field emitter
}

\author{
Chiwon Lee, ${ }^{1}$ Soichiro Tsujino, ${ }^{2, a)}$ and R. J. Dwayne Miller ${ }^{1,3, b)}$ \\ ${ }^{1}$ Max Planck Institute for the Structure and Dynamics of Matters, Luruper Chausee 149, 22607 Hamburg, \\ Germany \\ ${ }^{2}$ Laboratory for Micro and Nanotechnology, Photon Science Division, Paul Scherrer Institut, Villigen 5232, \\ Switzerland \\ ${ }^{3}$ Departments of Chemistry and Physics, University of Toronto, Toronto, M5S 3 H6 Ontario, Canada
}

(Received 26 March 2018; accepted 18 June 2018; published online 5 July 2018)

\begin{abstract}
We explore the spatial coherence of double-gate single nanotip field emitters by low-energy electron diffraction experiments in transmission mode. By producing collimated field emission pulses from the single nanotip cathode and irradiating a suspended monolayer graphene film without additional optics, we observed sharper and higher resolution Bragg diffraction spots than a previous experiment using a nanotip array cathode. In particular, we found complete conservation of the size and the shape of the diffraction spots with those of the incident beam on the sample. The result indicates that the transverse coherence of a nanofabricated double-gate single-tip emitter is much larger than a few nanometers as determined by the apparent diffraction spot size and overall spatial resolution of the observed diffraction pattern. Published by AIP Publishing.
\end{abstract}

https://doi.org/10.1063/1.5030889

The large scattering cross section of electrons makes electron diffraction experiments advantageous in determining atomic structures of small crystal samples that are difficult with X-rays. ${ }^{1}$ However, to provide the needed resolution, the transverse coherence length of the electron beam should exceed tens or hundreds of angstroms to analyze large-unit-cell organic or biological crystal samples. ${ }^{2-4}$ High-resolution electron microscopy can routinely achieve sub-angstrom resolution in small solid particles or nearatomic-resolution single-particle imaging for biological specimens, ${ }^{5,6}$ but only through the sacrifice of electron flux by magnifying and clipping the beam with an aperture. The development of ultrabright electron sources using cathodes based on metal nanotip emitters ${ }^{7-9}$ has been motivated by the desire to overcome the limit of the spatial and temporal resolution for time-resolved studies that are compromised or unattainable with low flux sources. ${ }^{3,4}$ The attainment of high field emission current densities of $10^{6}-10^{7} \mathrm{~A} / \mathrm{cm}^{2}$ within the desired narrow intrinsic energy spread of $\sim 0.2 \mathrm{eV}$ produced from a few nanometer nanotip apex (with even smaller virtual source size) is exceedingly hard to achieve with thermionic emitters or UV-excited photocathodes. ${ }^{10-13}$

In the literature, electron guns using etched-wire needleshaped field emitters have been reported. ${ }^{14-17}$ However, these sources suffer from the large geometrical divergence of the field emission beam because of the curved emitter surface, but not due to the intrinsic transverse velocity spread. For efficient use of the bunch charge and to utilize the beam with its intrinsic brightness, dedicated optics are normally required. In addition, the requirement of a high acceleration field to suppress the space-charge degradation of the beam brightness and coherence is often compromised when the

\footnotetext{
a) soichiro.tsujino@psi.ch

b) dwayne.miller@mpsd.mpg.de
}

acceleration field is coupled to the electron emission from nanotip sources. In contrast, irradiating samples with collimated field emission pulses without additional optics under the required high acceleration field is feasible with the double-gate nanotip emitter structure because of the on-chipintegrated beam collimator and the electrostatic shielding of the nanotip from the external field. ${ }^{18-20}$

In this letter, we study low-energy electron diffraction from suspended graphene to explore the transverse coherence property of double-gate field emitters. Compared to our recent experiment using a $10^{4}$-nanotip array (or field emitter array, FEA) double-gate emitter, ${ }^{12}$ we were able to achieve diffraction with improved spatial resolution and signal quality. We found a clear influence of the beam shape and the sample size on the Bragg diffraction spots from graphene. This indicates that the transverse coherence length of our single nanotip source is much larger than the value evaluated from the apparent Bragg spot size. In addition, close inspection of the Bragg diffraction intensity shows that the on-chip beam collimation not only reduces the beam divergence but significantly improve the wave front flatness.

We prepared the double-gate single nanotip emitter [Figs. 1(a) and 1(b)] with the same design and fabrication procedure reported previously; ${ }^{19-21}$ the emitter was a pyramidal shape molybdenum with the tip apex diameter of $10-20 \mathrm{~nm}$. The electron extraction gate $G_{e x t}$ and beam collimation gate $G_{c o l}$ layers, respectively, $500 \mathrm{~nm}$ - and $300 \mathrm{~nm}$-thick, were stacked on top of the emitter 1.2- $\mu$ m-thick insulating layers [see Fig. 1(b)]. The diameter of the $G_{e x t}$ and $G_{c o l}$ apertures were equal to $1.8 \mu \mathrm{m}$ and $5.5 \mu \mathrm{m}$, respectively, as measured by SEM [Fig. 1(a)]. To measure the transmission through and the Bragg diffraction from a suspended monolayer graphene sample, we loaded the emitter into the setup depicted in Fig. 2(a). The monolayer graphene sample was supported on a copper TEM grid $\left(\right.$ PELCO $\left.^{\circledR}\right)$. 
(a)

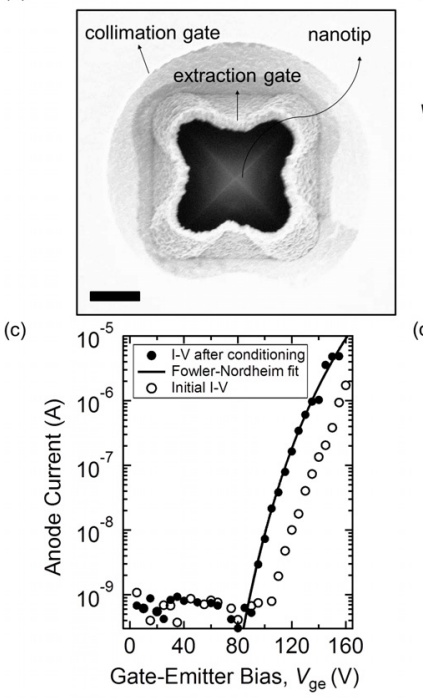

(b)

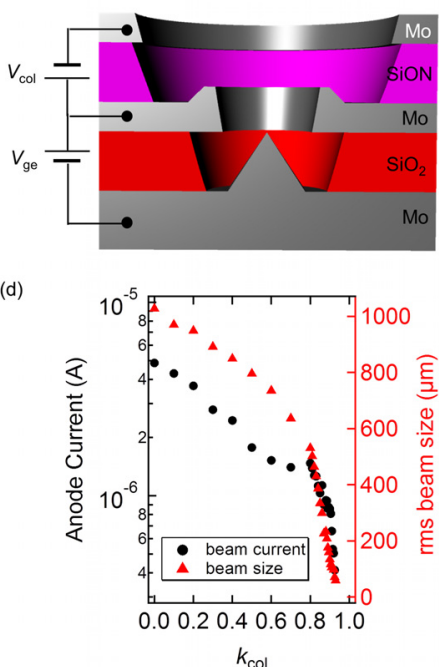

FIG. 1. (a) SEM image of the double-gate single nanotip field emitter device (top view). Scale bar is $1 \mu \mathrm{m}$. (b) Schematic illustration of the field emitter, cutout of the side view. Emitter base plate and collimation gate layer are electrically biased at negative potential with respect to the extraction gate biased at ground. $V_{g e}$ and $V_{c o l}$ refer to extraction gate-to-emitter voltage and collimation voltage, respectively. (c) $I-V$ of the device. Empty circles show the initial $I-V$, and the filled circles show the $I-V$ after 30 min of conditioning. The line superposed to the latter shows the fitting of the $I-V$ by the Fowler-Nordheim equation as described in the main text. (d) Beam current and the rms beam size variation depending on the collimation voltage. $k_{c o l}$ refers to the ratio $V_{c o l}$ to $V_{g e}$.

After we evacuated the experimental chamber down to $(1-1.4) \times 10^{-8}$ mbar, we first conditioned the emitter by repeatedly applying the electron extraction potential $V_{g e}$ to $G_{\text {ext }}$ with respect to the emitter substrate from $0 \mathrm{~V}$ to a certain value with the zero $V_{c o l}$, where we define the collimation potential $V_{c o l}$ as the potential applied to $G_{c o l}$ with respect to $G_{\text {ext }} . G_{\text {ext }}$ was connected to the ground potential in this and following measurements. The field emission current was measured by using the sample holder as the anode biased at $30 \mathrm{~V}$. We continued the conditioning for $30 \mathrm{~min}$ until the current-voltage characteristics $(I-V)$ became stable as shown in Fig. 1(c) by filled circles (the empty circles show the

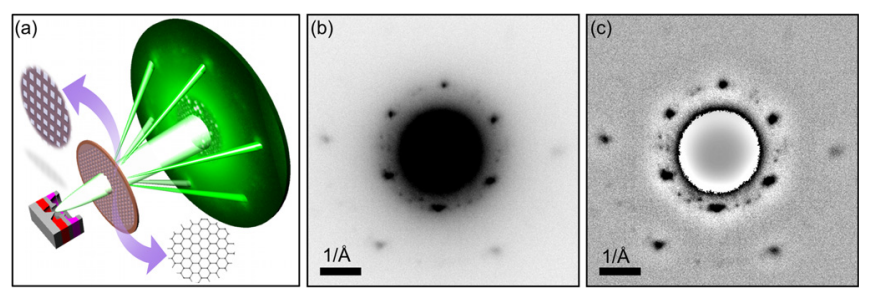

FIG. 2. (a) Schematic of the transmission low-energy electron diffraction experiment from a suspended monolayer of graphene. Beam size at the sample position is estimated by the projected shadow image of the TEM grid on which single graphene layer is covered with lacy carbon sheet. (b) Observed electron transmission image through a suspended monolayer of graphene for which the collimation potential of the double-gate single-nanotip field emitter was set at the maximally collimation condition $\left(k_{c o l}=0.92\right)$ with $V_{g e}$ $=80 \mathrm{~V}$. The hexagonal 1st order and the 2nd order Bragg reflection peaks are clearly observed. With the given electron beam wavelength at $900 \mathrm{eV}$ and the c-c bond length of graphene, the Bragg angle and the camera length are calculated to $8.26^{\circ}$ and $19.2 \mathrm{~mm}$, respectively. (c) High frequency filtered image of Fig. 2(b) to highlight the satellite Bragg diffraction spots closed to the 1 st order spots.

initial $I-V)$. After the conditioning, the emission current was equal to $4.86 \mu \mathrm{A}$ at $V_{g e}=155 \mathrm{~V}$. The leak current through the gate electrodes was several orders below that value. The $I-V$ fits well to a relation, $I=A_{F N}\left(V_{g e} / B_{F N}\right)^{2} \exp \left(-B_{F N} / V_{g e}\right)$, with the fitting parameters of $A_{F N}=20$ and $B_{F N}=1600$. Using these fitting parameters (in particular from $B_{F N}$ ), we estimated the electric field at the emitter tip apex $F_{\text {tip }}$ was equal to $6.31 \mathrm{~V} / \mathrm{nm}$ at $V_{g e}=155 \mathrm{~V}$ with the assumed value of the work function $\phi=4.5 \mathrm{eV}$ (for molybdenum). ${ }^{11}$

For the electron transmission and diffraction experiments, we fixed $V_{g e}=90 \mathrm{~V}$ with the corresponding zerocollimation potential emission current of $530 \mathrm{pA}$. To produce collimated field emission pulses, we applied the $V_{g e}$ pulse and the $V_{c o l}$ pulse synchronously. We applied $800 \mathrm{~V}$ to the sample. The field emission electron pulses were then accelerated at the extraction field of $\sim 0.4 \mathrm{MV} / \mathrm{m}$ in the gap between the cathode and the sample holder with the separation of $\sim 2 \mathrm{~mm}$, propagated through the $2 \mathrm{~mm}$-diameter-hole of the $3 \mathrm{~mm}$-thick sample holder, and irradiated the sample. The graphene sample was mounted on the electron detector side of the sample holder. The transmitted direct beam and the Bragg diffracted beams propagated approximately $20 \mathrm{~mm}$ to the electron detector. We applied $500 \mathrm{~V}$ at the entrance plane of the electron detector, $100 \mathrm{~V}$ to the MCP for the amplification, and $4.5 \mathrm{kV}$ to the phosphor screen for the electron detection. The beam images were subsequently captured by a CCD camera triggered synchronously with the gate pulses. To image the direct transmission beam, we applied 10- $\mu$ slong gate pulses. The beam size on the sample was evaluated by using the shadow of the TEM grid with the grid spacing of $85 \mu \mathrm{m}$ as the scale bar, as a function of $V_{c o l}$ [Fig. 1(d)], in which $V_{c o l}$ was specified by $k_{c o l}=\left|V_{c o l} / V_{g e}\right|$. The relationship between the emission current and $k_{\text {col }}$ estimated from the integrated image intensity is also shown in Fig. 1(d). We note that, due to the increased fraction of the TEM grid area as the beam spot size was reduced at larger $k_{c o l}$, this method systematically underestimates the current of the collimated beam. Nevertheless, the observation that the emission current was about $10 \%$ of the zero- $k_{c o l}$ value was consistent to previous experiments. ${ }^{20,21}$ At $k_{\text {col }}=0.925$, the rms (root mean square) beam size reached $59 \mu \mathrm{m}$. This was a factor of 17 smaller than that of zero $k_{c o l}$ beam. Beyond this $k_{c o l}$ value, the emission current was quickly quenched.

To study the electron diffraction from the graphene sample, we applied 900- $\mu$ s-long gate pulses at $V_{g e}=90 \mathrm{~V}$. As shown in Fig. 2(b), we were able to observe the hexagonally arranged clear 1st and the 2nd order diffraction spots from the graphene by irradiating a single collimated field emission pulse with $\sim 50 \mathrm{pA}$ current amplitude and $k_{\text {col }}=0.92$ (at $V_{g e}=90 \mathrm{~V}$ ) with $\sim 3 \times 10^{5}$ electrons in the pulse. The signal from the direct beam was saturated on the camera at this measurement condition.

To analyze the diffraction spots at high signal-to-noise ratio, we have digitally averaged 20 images repeatedly captured at the same condition and subtracted the background. In Fig. 3(a), we show the evolution of the direct beam (left panel) and diffraction spots (right panel) when $k_{c o l}$ was increased from 0.8 to 0.925 . The direct beams showed randomly distributed bright spots in addition to the shadow of the grid. We ascribe these bright spots to the 
(a)
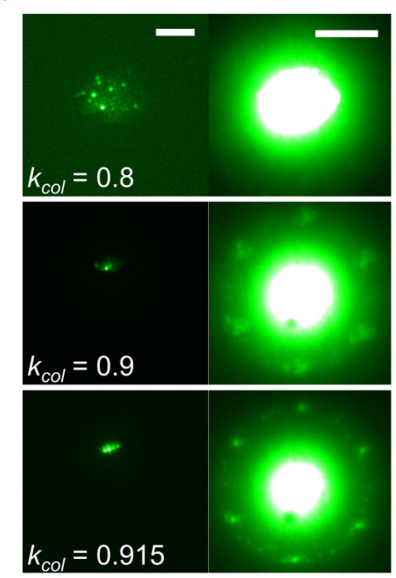
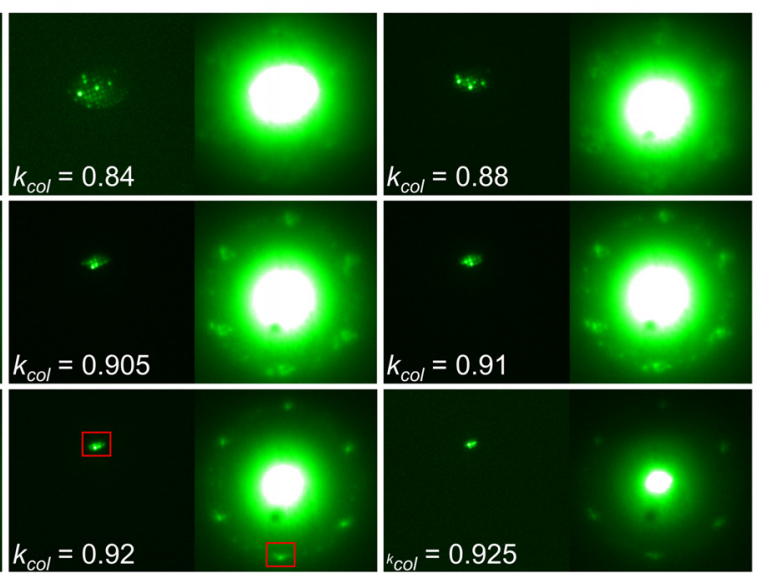

(b)

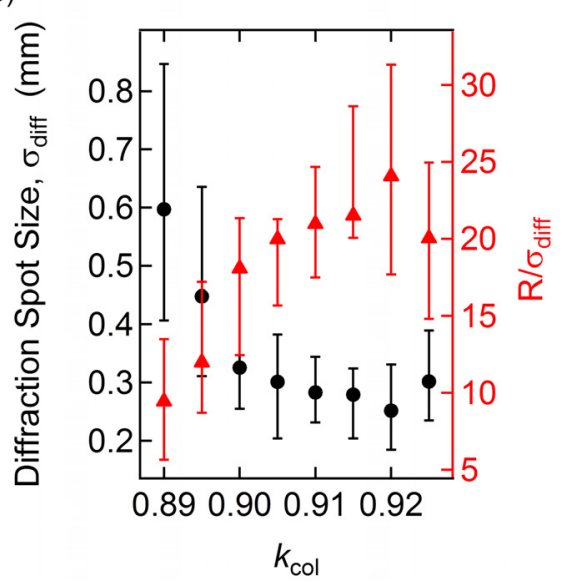

FIG. 3. (a) Selected image display of the direct (left panel) and the diffracted beam (right panel). Scale bar is $500 \mu \mathrm{m}$ and $5 \mathrm{~mm}$ on the respective beam image. For some $k_{c o l}$-value images, magnified views are shown in Fig. 4(a) for the direct beam and one of the diffraction spots for the parts indicated by the red box in the image of $k_{c o l}=0.92$. (b) Variation of the rms diffraction spot size $\left(\sigma_{\text {diff }}\right)$ and the ratio between beam center-to-diffraction spot distance and $\sigma_{\text {diff }}$ as a function of $k_{c o l}$.

non-uniformity of the sample, since these were not observed in the separate beam imaging experiment without the sample. Figure 3(a) shows that, when $k_{c o l}$ was increased from 0.8 to 0.925 and the beam spot size was reduced from $0.5 \mathrm{~mm}$ to $59 \mu \mathrm{m}$ [Fig. 1(d)], the probed area of the sample was reduced from $\sim 15$ grid to within approximately one grid zone. The center of the beam shifted upwards by a small amount at the same time, perhaps due to the non-uniformity of the beam acceleration or residual magnetic field. When $k_{c o l}$ was increased to 0.84 and the beam spot size was reduced by one third of the zero $k_{c o l}$ case [Fig. 1(d)], the hexagonal Bragg reflection spots became visible. Each diffraction spot emerged as a group of spots around the six-fold symmetric position. With the further increase of $k_{c o l}$ and the decrease of the beam spot [Fig. 1(d)], the Bragg reflection spots including the satellite spots became clearer and their overall $\mathrm{rms}$ radius, $\sigma_{\text {diff, }}$ became smaller [Fig. 3 (b)]. This is similar to that observed previously with FEA beams, ${ }^{12}$ but the present result obtained by using the single nanotip emitter exhibits higher spatial resolution. This is also indicated by the factor of $\sim 2$ larger $R / \sigma_{\text {diff }}$ ratio [Fig. 3(b)] observed here than the FEA experiment, where $R$ is the distance between the center of the direct beam to the center of the diffraction spots. The satellite spots with the same $R$ reflect the contribution of multiple graphene lattice domains at the large beam size.

When we analyze the main Bragg spot size by a Gaussian- and axial-wave model with a full consideration of the diffraction angle-dependent electron elastic scattering cross-section, we obtained the lower estimate transverse coherence length ${ }^{16}$ of $\sim 1 \mathrm{~nm}$, which is a few times smaller than the radius of the curvature of the nanotip apex. However, we consider that the spatial coherence length to be much larger than this value. This is on one hand because of the similarity of the Bragg diffraction spots with the direct beam shape (see below), and on the other hand, because of the observation of the satellite Bragg diffraction spots that are an order of magnitude smaller than the main diffraction spots [Fig. 2(c)], hence indicating a transverse coherence length of tens of nanometers.
When the spot size of the incident beam is small and the sample is uniform over the irradiated spot, the $R / \sigma_{\text {diff }}$ ratio can be a quantitative measure of the transverse coherence length of the incident beam. ${ }^{16}$ However, when the beam size is finite and the sample is not uniform, $\sigma_{\text {diff }}$ is rather determined by the beam size and the non-uniformity of the sample instead of the spatial coherence length and the $R / \sigma_{\text {diff }}$ ratio merely gives the lower estimate of the coherence length. From the comparison of the direct beam and the diffraction spots, we consider this applies to the present experiment: in Fig. 4(a), we show the magnified images of the direct beam and one of the 1st order diffraction spots (in the 6 o'clock direction). These were taken from the region indicated by the red boxes in Fig. 3(a) (see $k_{c o l}=0.92$ image). The strong similarity of the Bragg reflection spots and the direct beam shape is apparent.

Because of the highly coherent nature of the field emission beam, ${ }^{22}$ the transverse spread of the wave function of the field emission electron beam is much smaller than the incoherent beam case. The acceleration of the beam along the beam axis as in the present geometry should also have an effect in making the transverse spread of the wave function upon propagation narrower than the free-space propagation. However, for a field emitter with the apex radius of curvature of $5 \mathrm{~nm}$, the expected transverse spread is several microns even under the finite acceleration. ${ }^{23}$ This is consistent with our present conclusion that the transverse coherence length of the single-nanotip double-gate emitter is much larger than the value determined by the apparent Bragg diffraction spot size that might be larger than tens of nanometers as the small satellite Bragg diffraction spot size indicates. Further experimental characterization of the transverse coherence length of our nanotip emitters is of particular practical relevance, requiring experiments with large unit cell systems for calibration and determination of the upper limit to the coherence that will be most relevant to spatial resolving power of atomic structures.

We finally note the influence of the increased $k_{c o l}$ on the brightness of the diffraction spots observed in Fig. 4(a). In Fig. 4(b), the ratio of the brightness of the diffraction spot on that of the direct beam was summarized. When $k_{c o l}$ was 
(a)
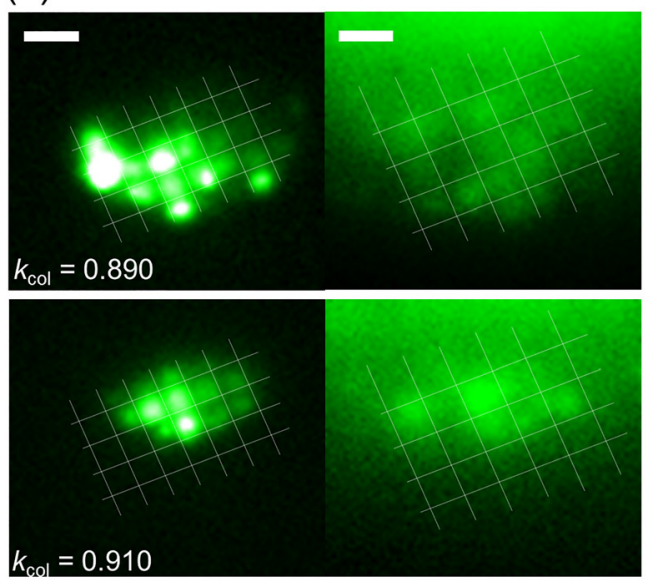
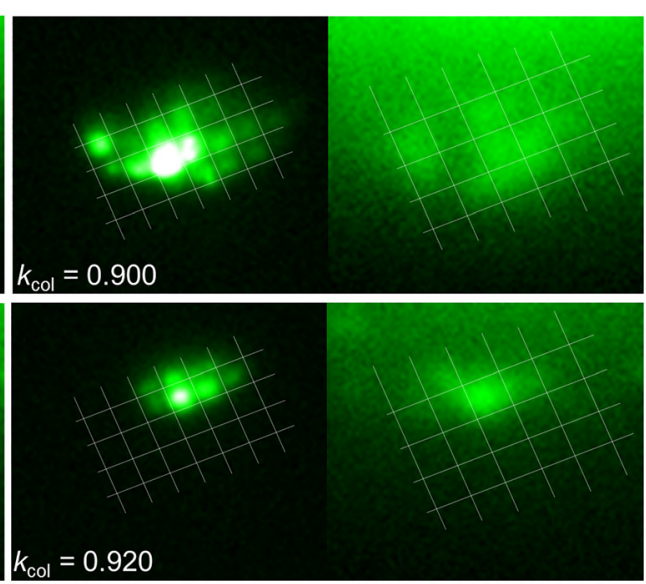

(b)

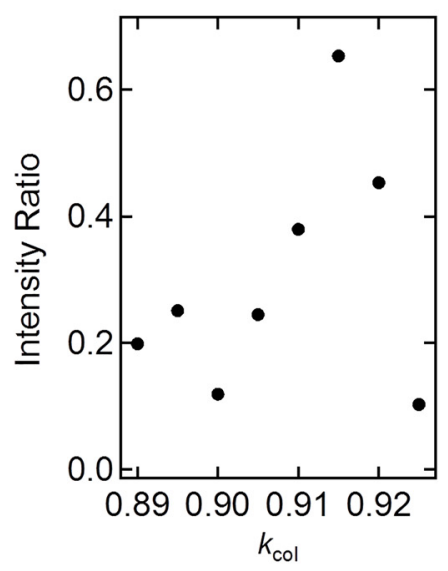

FIG. 4. (a) Magnified comparison of the direct beam spot and the diffraction spot at various $k_{c o l}$. Cropped and magnified region [indicated by red box in Fig. 3(a)] of the shadow image (left panel) and the diffraction spot at the 6 o'clock position in the diffraction patterns shown in Fig. 3 (right panel). The grid spacing is $85 \mu \mathrm{m}$ on the actual sample surface. The scale bars denoted on the images are $500 \mu \mathrm{m}$ indicating the size of the beam on the phosphor screen. (b) Variation of the brightness of the Bragg reflection spot to that of the direct beam with the increase of $k_{\text {col }}$ from 0.89 and 0.925 .

increased, the diffraction spot size decreased because of the decrease of the direct beam spot size, thereby increasing the spatial resolution of the electron probe. However, the additional increase in the brightness of the diffraction spot is governed by a separate effect. We ascribe this to the fact that the increasing $k_{c o l}$ is not only collimating the otherwise diverging electron trajectories emitted from the nanotip apex but also flattening the electron wave front, hence substantially reducing the beam-front-dependent angular dispersion of the Bragg diffraction direction. Figure 4(b) shows that the wave front was most flat when $k_{c o l}$ was $0.915-0.920$, and its curvature increased at higher $k_{c o l}$ due to over focusing, even though the beam spot was the smallest at $k_{\text {col }}=0.925$. This highlights the unique characteristic of our double-gate nanotip emitter that allowed for detecting sharp diffraction images without external optics.

In summary, we demonstrated that the double-gate single nanotip emitter is capable of producing sharp Bragg diffraction from a suspended monolayer graphene sample. By using the on-chip beam collimator, we were able to generate electron pulses that are not only collimated but also spatially coherent with coherence lengths greater than tens of nanometers, allowing for the low-energy transmission diffraction experiment using a minimal experimental setup without additional electron optical elements. Analysis of the recorded diffraction and direct beam images and the observation of small satellite diffraction spots clearly showed that shape and pattern of the diffraction spots are dependent both on the angular divergence and size of the electron beam that are controlled by the on-chip collimator of our device.

See supplementary material for the entire collection of the direct and diffracted beam images (supplementary Fig. S1) and the derivation of transverse coherence length of collimated electron beam.

This work was funded by the Max Planck Society. C.L. is grateful to Dr. A. Marx, Dr. S. Hayes, and Dr. K. Siddiqui for the helpful discussion on the diffraction data analysis.
The authors acknowledge Dr. V. Guzenko, J. Lehmann, Dr. P. das Kanungo, and K. Vogelsang for their support for the fabrication of the double-gate nanotip devices at the Paul Scherrer Institut (PSI). The work was also partially supported by the SwissFEL project at PSI.

${ }^{1}$ R. Henderson, Q. Rev. Biophys. 28(2), 171 (1995).

${ }^{2}$ P. Baum, Chem. Phys. 423, 55 (2013).

${ }^{3}$ R. J. D. Miller, Science 343(6175), 1108 (2014).

${ }^{4}$ A. A. Ischenko, P. M. Weber, and R. J. D. Miller, Chem. Rev. 117, 11066 (2017).

${ }^{5}$ Y. Fujiyoshi, Adv. Biophys. 35, 25 (1998).

${ }^{6}$ J. C. H. Spence, High-Resolution Electron Microscopy (OUP Oxford, 2013).

${ }^{7}$ P. Hommelhoff, Y. Sortais, A. Aghajani-Talesh, and M. A. Kasevich, Phys. Rev. Lett. 96(7), 077401 (2006).

${ }^{8}$ C. Ropers, D. R. Solli, C. P. Schulz, C. Lienau, and T. Elsaesser, Phys. Rev. Lett. 98(4), 043907 (2007).

${ }^{9}$ D. Ehberger, J. Hammer, M. Eisele, M. Krüger, J. Noe, A. Högele, and P. Hommelhoff, Phys. Rev. Lett. 114(22), 227601 (2015).

${ }^{10}$ J. W. Gadzuk and E. W. Plummer, Rev. Mod. Phys. 45(3), 487 (1973).

${ }^{11}$ R. G. Forbes and J. H. B. Diane, Proc. R. Soc. London, Ser. A 463, 2907 (2007).

${ }^{12}$ S. Tsujino, P. D. Kanungo, M. Monshipouri, C. Lee, and R. J. D. Miller, Nat. Commun. 7, 13976 (2016).

${ }^{13}$ A. Feist, N. Bach, N. R. D. Silva, T. Danz, M. Möller, K. E. Priebe, T. Domröse, J. G. Gatzmann, S. Rost, and J. Schauss, Ultramicroscopy 176, 63 (2017).

${ }^{14}$ A. Paarmann, M. Gulde, M. Müller, S. Schäfer, S. Schweda, M. Maiti, C. Xu, T. Hohage, F. Schenk, and C. Ropers, J. Appl. Phys. 112(11), 113109 (2012).

${ }^{15}$ M. Gulde, S. Schweda, G. Storeck, M. Maiti, H. K. Yu, A. M. Wodtke, S. Schäfer, and C. Ropers, Science 345(6193), 200 (2014).

${ }^{16}$ M. Muller, A. Paarmann, and R. Ernstorfer, Nat. Commun. 5, 5292 (2014).

${ }^{17}$ G. Storeck, S. Vogelgesang, M. Sivis, S. Schäfer, and C. Ropers, Struct. Dyn. 4(4), 044024 (2017).

${ }^{18}$ P. Helfenstein, V. A. Guzenko, H. W. Fink, and S. Tsujino, J. Appl. Phys. 113(4), 043306 (2013).

${ }^{19}$ E. Kirk, S. Tsujino, T. Vogel, K. Jefimovs, J. Gobrecht, and A. Wrulich, J. Vac. Sci. Technol., B: Microelectron. Nanometer Struct. Process., Meas., Phenom. 27(4), 1813 (2009).

${ }^{20}$ C. Lee, P. D. Kanungo, V. Guzenko, P. Hefenstein, R. J. D. Miller, and S. Tsujino, J. Vac. Sci. Technol., B 33(3), 03C111 (2015).

${ }^{21}$ P. Helfenstein, E. Kirk, K. Jefimovs, T. Vogel, C. Escher, H. W. Fink, and S. Tsujino, Appl. Phys. Lett. 98(6), 061502 (2011).

${ }^{22}$ T. Latychevskaia, Ultramicroscopy 175, 121 (2017).

${ }^{23}$ S. Tsujino, "Transverse structure of the wave function of field emission electron beam determined by intrinsic transverse energy," J. Appl. Phys. (submitted). 